15,11

\title{
Индуцированный магнитным полем ориентационный переход в геликоидальном жидкокристаллическом антиферромагнетике
}

\author{
(C) А.Н. Захлевных, К.В. Кузнецова \\ Пермский государственный национальный исследовательский университет, \\ Пермь, Россия \\ E-mail: anz@psu.ru
}

(Поступила в Редакцию 14 апреля 2016 г.)

\begin{abstract}
Теоретически изучен индуцированный магнитным полем ориентационный переход в геликоидальных жидкокристаллических антиферромагнетиках - компенсированных суспензиях магнитных наночастиц в холестерических жидких кристаллах. Исследовано раскручивание спиральной структуры и поведение средней намагниченности как функций напряженности поля и материальных параметров. Показано, что в раскрученной полем ферронематической фазе магнитные подсистемы не полностью компенсированы, и ферронематическая фаза является ферримагнитной.
\end{abstract}

Работа выполнена при поддержке Российского фонда фундаментальных исследований (грант 16-42-590539).

\section{1. Введение}

Среди различных типов жидких кристаллов (ЖК) наиболее интересными свойствами обладают холестерические жидкие кристаллы (ХЖК). Они имеют спиральную надмолекулярную структуру и, вследствие этого, замечательные оптические свойства, позволяющие отнести их к классу фотонных кристаллов [1,2]. Суспензии магнитных наночастиц, приготовленные на их основе, получили название феррохолестериков $(\Phi \mathrm{X})$ или геликоидальных ферромагнитных жидких кристаллов (ФМ ЖК). В таких системах имеется ориентационная связь между длинными осями анизометричных частиц и ХЖК-матрицей, поэтому вектор намагниченности спирально закручен в пространстве. В этом смысле ФХ представляет собой жидкокристаллический аналог геликоидальных ферромагнетиков. В отличие от последних, магнитные частицы в $\Phi Х$ имеют возможность пространственного перемещения, мигрируя в те области образца, где минимальна их магнитная и ориентационная энергия (так называемый эффект сегрегации [3]). Феррохолестерики привлекательны открывающейся в них возможностью посредством слабых внешних магнитных полей изменять спиральность образуемой структуры и, тем самым, легко модулировать спектральный состав отраженного света. Теоретические и экспериментальные исследования физических явлений в геликоидальных ФМ ЖК вызывают в последние годы большой научный интерес [4-18].

Магнитное поле, приложенное перпендикулярно оси спиральной структуры $\Phi \mathrm{X}$, воздействует как на магнитные моменты феррочастиц (дипольный механизм), так и на диамагнитную холестерическую матрицу (квадрупольный механизм) и вызывает ее раскручивание (фазовый переход феррохолестерик-ферронематик $[4,6])$. Конкуренция энергии, описываемой градиентными инвариантами Лифшица, которая стремится произвести модуляцию структуры $\Phi \mathrm{X}$, и магнитной энергии ори- ентационных механизмов дипольного или квадрупольного типа, стремящейся создать ферронематическую фазу с однородной ориентацией директора, приводит к тому, что в окрестности этого перехода феррохолестерик описывается с помощью решетки солитонов (доменных стенок между доменами ферронематической фазы с разным направлением директора), вставленных в ферронематическую фазу. Типы возникающих доменных структур оказываются различными в области преобладающего влияния дипольного, либо квадрупольного механизма [5]. В отличие от геликоидального ферромагнетика с одноосной магнитной анизотропией в базисной плоскости (см., например, [19]), в феррохолестерике процесс раскручивания спиральной структуры осложняется тем, что роль оси анизотропии играет директор ХЖК-матрицы, который сам ориентируется магнитным или электрическим полем, и эффектом сегрегации магнитных частиц [3]. Диамагнитная анизотропия ХЖК-матрицы (положительная или отрицательная), намагниченность магнитных частиц, их пространственное распределение в образце и энергия сцепления молекул ХЖК с поверхностью магнитных частиц сильно влияют на структуру доменных стенок в раскрученных полем $\Phi Х$ По этим причинам поведение $\Phi Х$ в поле оказывается намного сложнее и разнообразнее, чем поведение твердых геликоидальных ферромагнетиков.

В зависимости от способа приготовления ФМ ЖК может быть намагниченным или компенсированным [3]. В намагниченных суспензиях магнитные моменты феррочастиц в каждой точке образца сонаправлены (жидкокристаллический аналог ферромагнетика), поэтому магнитная восприимчивость намагниченного ФМ ЖК существенно выше, чем у чистых ЖК. Вследствие этого, а также, благодаря ориентационной связи анизометричных частиц с упорядоченной ЖК-матрицей, поля переориентации ФМ ЖК снижаются до десятков и единиц эрстед, т.е. как минимум на два порядка по сравнению 
с чистыми жидкими кристаллами. В компенсированных ФМ ЖК имеются равные доли магнитной примеси с противоположно ориентированными магнитными моментами, так что в отсутствие магнитного поля компенсированный ФМ ЖК представляет собой жидкокристаллический аналог антиферромагнетика. В большинстве имеющихся теоретических работ рассматривались намагниченные ФМ ЖК, то есть предполагалось, что ФМ ЖК обладает начальной (спонтанной) намагниченностью (см., напр., обзор [20]). Между тем, если при изготовлении суспензии не принимать специальных мер для ее принудительного намагничивания, то ФМ ЖК будет находиться в компенсированном состоянии. Поведение компенсированного ФМ ЖК в магнитном поле, т.е. его магнитные, ориентационные и оптические свойства существенно отличаются от поведения намагниченной суспензии. Такие отличия показаны в работах [21-23], в которых построена теория индуцированных магнитным полем ориентационных переходов в компенсированных ФМ ЖК на основе нематического ЖК.

В настоящей работе рассматриваются индуцированные магнитным полем фазовые переходы в так называемых компенсированных феррохолестериках геликоидальных антиферромагнитных жидких кристаллах (АФМ ЖК).

\section{2. Свободная энергия и уравнения равновесия}

Как известно [1], холестерические жидкие кристаллы с положительной анизотропией $\chi_{a}$ диамагнитной восприимчивости претерпевают переход в нематическое состояние под действием магнитного поля $\mathbf{H}$, перпендикулярного оси спиральной структуры ХЖК. Критическое поле такого перехода

$$
H_{c}=\frac{\pi}{2} q_{0} \sqrt{\frac{K_{22}}{\chi_{a}}}
$$

$\left(q_{0}-\right.$ волновое число невозмущенной полем спирали, $K_{22}$ - модуль кручения ХЖК) в типичном ХЖК довольно велико (порядка $10^{3}-10^{4} \mathrm{Oe}$ ) вследствие малости $\chi_{a}$ $\left(\sim 10^{-6} \div 10^{-7}\right)$. С целью снижения величин управляющих магнитных полей Брошар и де Жен [3] предложили внедрить магнитные частицы в жидкокристаллическую матрицу. Такие магнитные жидкости на основе ХЖК получили название феррохолестериков. Если магнитные частицы имеют анизометричную форму (например, иглообразную), упругие взаимодействия вызывают их ориентацию в жидкокристаллической матрице. Такие суспензии, сохраняя все особенности ориентационного поведения ХЖК, имеют магнитную восприимчивость на несколько порядков большую, чем чистые ХЖК, что позволяет управлять их текстурой относительно слабым магнитным полем. Вследствие спиральной структуры ХЖК-матрицы вектор намагниченности ФХ закручен в пространстве вместе с директором $\mathbf{n}$ матрицы и в этом отношении ФХ являются жидкокристаллическими аналогами геликоидальных ферромагнетиков. В работе рассматриваются ориентационные и магнитные свойства компенсированных феррохолестериков (АФМ ЖК) в магнитном поле, перпендикулярном оси спирали. Будем полагать, что твердая фаза ФХ состоит из однодоменных иглообразных частиц ферромагнетика длиной $l \gg a$ и диаметром $d \sim l / 10$, где $a$ - длина молекулы ХЖК. Столь сильная анизометричность частиц приводит к жесткой связи магнитного момента с телом частицы. Обработка частиц поверхностно-активными веществами обеспечивает ориентационную связь их длинных осей с локальным направлением директора $\mathbf{n}$ холестерика. Однородное магнитное поле Н вызывает поворот феррочастиц (дипольный механизм) и, тем самым, искажения поля директора матрицы. Такая ситуация соответствует так называемому коллективному поведению $Ф Х$, при котором концентрация магнитных зерен должна превышать некоторое критическое значение, определяемое характерным размером образца [3]. Меньшие концентрации магнитной фазы приводят лишь к локальным искажениям поля директора вблизи каждой частицы независимо от других.

Таким образом, имеется два ориентационных механизма влияния магнитного поля на ФХ: дипольный (ферромагнитный), обусловленный взаимодействием магнитных моментов феррочастиц с полем, и квадрупольный (диамагнитный), связанный с воздействием поля на анизотропную ХЖК-матрицу, в то время как в чистых ХЖК присутствует только квадрупольный механизм. Дипольный механизм линеен по полю, и именно он определяет поведение ФХ в слабых полях, что приводит к существенному снижению управляющих магнитных полей.

Ниже мы рассматриваем компенсированный ФХ с жестким планарным сцеплением магнитных частиц с матрицей, при котором намагниченность ФХ параллельна директору. В этом случае как дипольный, так и квадрупольный механизмы способствуют раскручиванию спиральной структуры, что приводит к снижению порогового поля перехода феррохолестерик-ферронематик по сравнению с чистым ХЖК.

Рассмотрим ориентационные и магнитные свойства компенсированного ФХ с положительной диамагнитной анизотропией $\chi_{a}$ в магнитном поле, перпендикулярном оси спирали. Равновесная конфигурация директора и намагниченности $\Phi Х$ определяется из условий минимума полной свободной энергии $\mathscr{F}=\int F d V$, где объемная плотность $F$ свободной энергии имеет вид [3]

$$
\begin{gathered}
F=F_{1}+F_{2}+F_{3}+F_{4}, \\
F_{1}=\frac{K_{11}}{2}(\operatorname{div} \mathbf{n})^{2}+\frac{K_{22}}{2}\left(\mathbf{n} \operatorname{rot} \mathbf{n}+q_{0}\right)^{2}+\frac{K_{33}}{2}(\mathbf{n} \times \operatorname{rot} \mathbf{n})^{2}, \\
F_{2}=-\frac{\chi_{a}}{2}(\mathbf{n} \mathbf{H})^{2}, \\
F_{3}=-M_{s}\left(f_{+}-f_{-}\right) \mathbf{m} \mathbf{H}, \\
F_{4}=\frac{k_{B} T}{v}\left[f_{+} \ln f_{+}+f_{-} \ln f_{-}\right] .
\end{gathered}
$$

Здесь $K_{11}, K_{22}, K_{33}$ - модули ориентационной упругости ХЖК (константы Франка), $q_{0}$ - волновое число 
спиральной структуры, $p_{0}=2 \pi / q_{0}-$ шаг спиральной структуры в отсутствие поля, $\chi_{a}=\chi_{\|}-\chi_{\perp}-$ анизотропия диамагнитной восприимчивости ХЖК, $M_{s}-$ намагниченность насыщения материала магнитных частиц, $v$ - объем частицы, $f_{+}$и $f_{-}$- объемные доли магнитных частиц в суспензии, направленных, соответственно, вдоль и против директора, $\mathbf{m}$ - единичный вектор намагниченности. Слагаемое $F_{1}$ представляет собой объемную плотность энергии ориентационно-упругих деформаций директора $\mathbf{n}, F_{2}$ описывает плотность энергии взаимодействия диамагнитной ХЖК-матрицы с магнитным полем Н, $F_{3}$ - плотность энергии взаимодействия магнитных частиц с полем. Последнее слагаемое $F_{4}$ дает вклад энтропии смешения идеального раствора частиц в свободную энергию. Мы полагаем среднюю объемную долю частиц в суспензии $f_{0}=N v / V \ll 1$, поэтому магнитными диполь-дипольными взаимодействиями частиц можно пренебречь (здесь $N$ - число частиц в суспензии, $V$ - объем образца).

Будем полагать, что $\chi_{a}>0$ и в отсутствие внешнего магнитного поля магнитные частицы внедрены в ХЖК-матрицу планарно $(\mathbf{m} \| \mathbf{n})$, а сцепление частиц с директором абсолютно жесткое $(\mathbf{m} \equiv \mathbf{n})$ и $\Phi \mathrm{X}$ является компенсированным, т.е. феррочастицы с магнитными моментами, направленными параллельно $\left(f_{+}\right)$ и антипараллельно $\left(f_{-}\right)$директору $\mathbf{n}$, имеют равные объемные доли $\left(f_{+}=f_{-}=f_{0} / 2\right)$, и, следовательно, их магнитные моменты вращаются вместе с директором $\mathbf{n}=\mathbf{n}_{0}=\left(\cos q_{0} z, \sin q_{0} z, 0\right)$, формируя спираль $\Phi \mathbf{X}$ шагом $p_{0}=2 \pi / q_{0}$. Тем самым, результирующая намагниченность ФХ закручена в пространстве по типу геликоидального антиферромагнетика.

Направим теперь внешнее магнитное поле $\mathbf{H}=(0, H, 0)$ поперек оси спирали ФХ. Магнитное поле вызывает искажения геликоидальной структуры $\Phi Х$, и зависимость компонент директора от координаты $z$ перестает быть гармонической

$$
\mathbf{n}=(\cos \varphi(z, H), \sin \varphi(z, H), 0) .
$$

Здесь $\varphi(z, H)$ - угол поворота директора и намагниченности, отсчитываемый от оси $x$. Таким образом, при $H \neq 0$ директор неравномерно вращается при движении вдоль оси $z$, чаще занимая направление вдоль приложенного поля.

Условие постоянства числа магнитных частиц в суспензии записывается в виде

$$
\int\left(f_{+}+f_{-}\right) d V=N v
$$

Подставим (3) в (2)

$$
\begin{aligned}
F & =\frac{K_{22}}{2}\left(\frac{d \varphi}{d z}-q_{0}\right)^{2}-\frac{\chi_{a} H^{2}}{2} \sin ^{2} \varphi \\
& -M_{s}\left(f_{+}-f_{-}\right) H \sin \varphi+\frac{k_{B} T}{v}\left(f_{+} \ln f_{+}+f_{-} \ln f_{-}\right) .
\end{aligned}
$$

Далее удобно перейти к безразмерным величинам. Для этого выберем в качестве единицы длины величину $q_{0}^{-1}$, тогда безразмерная координата $\tilde{z}=q_{0} z$, а в качестве единицы напряженности магнитного поля величину $H_{q}=q_{0} \sqrt{K_{22} / \chi_{a}}$, имеющую порядок поля перехода в нематическую фазу в чистом ХЖК [1], а также в ФХ, если квадрупольный механизм воздействия на систему преобладает над дипольным [4]. Безразмерную напряженность поля определим соотношением $h=H / H_{q}$. Если же преобладает дипольный механизм взаимодействия, то упругий $\left(F_{1}\right)$ - и дипольный $\left(F_{3}\right)$-вклады в объемную плотность свободной энергии $F$ оказываются одного порядка при $H=H_{d}$, где $H_{d}=K_{22} q_{0}^{2} /\left(M_{s} f_{0}\right)-$ характерное поле перехода в ФН-фазу при преобладании дипольного механизма [4].

Приведенные объемные доли $g_{ \pm}$частиц определим соотношением $f_{ \pm}=f_{0} g_{ \pm}$, тогда условие (4) запишется в виде

$$
\frac{1}{V} \int\left(g_{+}+g_{-}\right) d V=1 .
$$

В этих переменных безразмерная плотность $\tilde{F}=F /\left(K_{22} q_{0}^{2}\right) \quad$ свободной энергии (5) принимает вид

$$
\begin{aligned}
\tilde{F} & =\frac{1}{2}\left(\frac{d \varphi}{d \tilde{z}}-1\right)^{2}-\frac{1}{2} h^{2} \sin ^{2} \varphi \\
& -\xi h\left(g_{+}-g_{-}\right) \sin \varphi+\kappa\left(g_{+} \ln g_{+}+g_{-} \ln g_{-}\right) .
\end{aligned}
$$

Здесь введены обозначения для безразмерных параметров

$$
\xi \equiv \frac{M_{s} f_{0}}{q_{0} \sqrt{K_{22} \chi_{a}}}, \quad \kappa \equiv \frac{k_{B} T f_{0}}{v K_{22} q_{0}^{2}} .
$$

Параметр $\xi=H_{q} / H_{d}$ характеризует режим раскручивания спирали: при $\xi \gg 1$ (т.е. $H_{q} \gg H_{d}$ ) раскручивание спиральной структуры вызывается дипольным механизмом, а при $\xi \ll 1-$ квадрупольным. Параметр $\kappa$ отвечает за эффект сегрегации [3]: магнитное поле Н индуцирует перераспределение магнитной примеси по объему ФХ, вызывая миграцию магнитных частиц в те области спиральной структуры $Ф$, где направления $\mathbf{H}$ и $\mathbf{m}$ близки. В результате жесткой связи $(\mathbf{m} \| \mathbf{n})$ между ориентациями частиц и директором создается ориентационная деформация в области с характерным размером $\lambda$. В равновесном состоянии $\lambda$ определяется из условия баланса плотности упругой энергии $F_{1} \approx K_{22} / \lambda^{2}$ и энтропийного вклада $F_{4} \approx f_{0} k_{B} T / v$. В результате для характерного масштаба области, где имеет место концентрационное расслоение, находим $\lambda=\left(K_{22} v / f_{0} k_{B} T\right)^{1 / 2}$. Как видно из (8), параметр $\kappa=\left(\lambda q_{0}\right)^{-2}$ представляет собой как раз квадрат отношения двух характерных длин: невозмущенного полем шага спирали $p_{0}=2 \pi / q_{0}$ и сегрегационной длины $\lambda$. При $\kappa \gg 1$ эффект сегрегации магнитных частиц оказывается несущественным, т.к. характерный размер области, где имеет место концентрационное перераспределение, становится малым по сравнению с шагом спирали $\left(p_{0} \gg \lambda\right)$. В случае 
$\kappa \leq 1$ перераспределение частиц по периоду спирали становится существенным.

Поскольку $g+$ и $g$ - зависят только от $z$, то условие (6) примет вид

$$
\frac{1}{\tilde{p}} \int_{0}^{\tilde{p}}\left(g_{+}+g_{-}\right) d \tilde{z}=1
$$

где $\tilde{p}$ - шаг спирали в поле.

Равновесные состояния ФХ определяются условиями минимума полной свободной энергии

$$
\tilde{\mathscr{F}} \equiv \frac{\mathscr{F}}{V K_{22} q_{0}^{2}}=\frac{1}{\tilde{p}} \int_{0}^{\tilde{p}} \tilde{F} d \tilde{z}
$$

которая представляет собой функционал относительно $\varphi(z), g_{+}(z)$ и $g_{-}(z)$. Здесь $\tilde{\mathscr{F}}-$ безразмерная плотность полной свободной энергии. Минимизируя (10) по $\varphi(z)$, получим

$$
\frac{d^{2} \varphi}{d \tilde{z}^{2}}=-\frac{1}{2} h^{2} \sin 2 \varphi-\xi h\left(g_{+}-g_{-}\right) \cos \varphi .
$$

Минимизация по $g_{ \pm}(z)$ проводится с дополнительным условием (9), учитываемым методом множителей Лагранжа, и дает концентрационные распределения частиц в двух магнитных подсистемах

$$
g_{ \pm}(\tilde{z})=Q \exp \left\{ \pm \frac{\xi h}{\kappa} \sin \varphi(\tilde{z})\right\}
$$

где нормировочная константа $Q$ определяется из условия (9):

$$
Q^{-1}=\frac{2}{\tilde{p}} \int_{0}^{\tilde{p}} \cosh \left\{\frac{\xi h}{\kappa} \sin \varphi(\tilde{z})\right\} d \tilde{z}
$$

Соотношение (12) описывает эффект сегрегации дисперсной фазы [3]: магнитные частицы мигрируют в те области спиральной структуры ФХ, в которых минимальна их энергия в поле $\mathbf{H}$.

Уравнение (11) можно проинтегрировать путем умножения на $d \varphi / d \tilde{z}$, тогда получим

$$
\frac{d \varphi}{d \tilde{z}}=\sqrt{A(\varphi)}
$$

где введено обозначение:

$$
A(\varphi) \equiv C-h^{2} \sin ^{2} \varphi-2 \kappa\left(g_{+}+g_{-}\right) .
$$

Здесь $C$ - константа интегрирования. Она находится из условия минимума полной свободной энергии $\mathscr{F}$. Мы выбрали знак плюс перед корнем в (14), полагая, что $d \varphi / d \tilde{z}>0$, т.е. угол поворота директора и намагниченности растет с ростом $z$ (вращение против часовой стрелки). Напомним, что в отсутствие поля $d \varphi / d z=q_{0}>0$.
Полагая, что начало координат выбрано так, что $\varphi(0)=0$, можно проинтегрировать уравнение $(14)$

$$
\tilde{z}=\int_{0}^{\varphi(\tilde{z})} \frac{d \varphi}{\sqrt{A(\varphi)}}
$$

Это уравнение определяет неявную функцию $\varphi(\tilde{z}, h)$ и, тем самым, согласно (3) - ориентацию директора и намагниченности. Шагу спирали $p$ отвечает поворот директора на $2 \pi$, поэтому интегрируя (14) по $\tilde{z}$ от нуля до $\tilde{p}$, находим уравнение для шага спирали в поле:

$$
\tilde{p}=\int_{0}^{2 \pi} \frac{d \varphi}{\sqrt{A(\varphi)}}
$$

Далее с помощью (7) и (14) вычисляем полную свободную энергию (10)

$$
\tilde{\mathscr{F}}=\frac{1}{\tilde{p}} \int_{0}^{2 \pi}[\sqrt{A(\varphi)}-2 \pi] d \varphi+\frac{1-C}{2}+\kappa \ln (e Q),
$$

где $e-$ основание натуральных логарифмов. Уравнение для константы $C$ находим из условия минимума свободной энергии (18)

$$
\int_{0}^{2 \pi} \sqrt{A(\varphi)} d \varphi=2 \pi
$$

С учетом (19) безразмерная плотность полной свободной энергии (18) становится равной

$$
\tilde{\mathscr{F}}=\frac{1-C}{2}+\kappa \ln (e Q) .
$$

Перепишем выражение (13) в удобном для расчетов виде, перейдя к интегрированию по углу $\varphi$ с помощью уравнения (14)

$$
Q^{-1}=\frac{2}{\tilde{p}} \int_{0}^{2 \pi} \cosh \left\{\frac{\xi h}{\kappa} \sin \varphi(\tilde{z})\right\} \frac{d \varphi}{\sqrt{A(\varphi)}} .
$$

В слабых полях $\left(h \ll h_{c}\right.$, где $h_{c}-$ поле перехода в $\Phi Н$ фазу) зависимость шага спирали от напряженности поля (17) можно представить в виде

$$
\tilde{p}=\tilde{p}_{0}\left[1+\frac{\xi^{2} h^{2}}{2 \kappa}\right]
$$

т.е. шаг спирали растет с ростом поля быстрее, чем в чистом ХЖК, для которого $\tilde{p} / \tilde{p}_{0}=1+$ const $\cdot h^{4}[1]$. Это связано с тем, что в ФХ слабое поле воздействует в первую очередь на магнитные частицы, а не на 
ХЖК-матрицу, имеющую малую магнитную восприимчивость. Заметим еще, что в некомпенсированном ФХ шаг спирали в слабых полях ведет себя по закону [4]

$$
\tilde{p}=\tilde{p}_{0}\left[1+\frac{\xi^{2} h^{2}}{2}\right]
$$

т. е. не зависит от $\kappa$.

Определим теперь магнитные характеристики. В условиях сильной связи ориентация магнитных частиц и ХЖК намагниченность $\mathscr{M}=M_{s} f_{0}\left(g_{+}-g_{-}\right) \mathbf{n}$ феррохолестерика параллельна локальному директору $\mathbf{n}$. C учетом соотношений (3) и (12) приведенная намагниченность $\mathbf{M}=\mathscr{M} /\left(M_{s} f_{0}\right)$ определяется комбинацией ориентационного $\varphi(z)$ и концентрационного $g_{ \pm}(z)$ распределений

$$
\mathbf{M}=2 \mathbf{n} Q \sinh \left\{\frac{\xi h}{\kappa} \sin \varphi\right\} .
$$

Это соотношение позволяет найти усредненные по периоду спирали компоненты вектора приведенной намагниченности

$$
\langle\mathbf{M}\rangle=\frac{1}{p} \int_{0}^{2 \pi} \mathbf{M} d z
$$

Переходя в (25) к интегрированию по $\varphi$ с помощью (14), получаем

$$
\begin{gathered}
\left\langle M_{x}\right\rangle=\left\langle M_{z}\right\rangle=0, \\
\left\langle M_{y}\right\rangle=\frac{2 Q}{\tilde{p}} \int_{0}^{2 \pi} \sin \varphi \sinh \left\{\frac{\xi h}{\kappa} \sin \varphi\right\} A^{-1 / 2}(\varphi) d \varphi .
\end{gathered}
$$

Теперь система уравнений (3), (12), (16), (17), (19), (21) и (26) замкнута и позволяет определить ориентационные и магнитные характеристики $\Phi \mathrm{X}$.

\section{3. Критическое поле}

Найдем поле перехода в ФН-фазу. Для этого вернемся к формуле (7). Если феррохолестерик раскручен полем и произошел переход в однородную ( $\varphi$ не зависит от $\tilde{z}$ ) ферронематическую фазу, то безразмерная плотность полной свободной энергии ФН-фазы принимает вид

$$
\begin{aligned}
\tilde{\mathscr{F}}_{0}= & \frac{1}{2}-\frac{1}{2} h^{2} \sin ^{2} \varphi_{0}-\xi\left(g_{+}-g_{-}\right) h \sin \varphi_{0} \\
& +\kappa\left(g_{+} \ln g_{+}+g_{-} \ln g_{-}\right) .
\end{aligned}
$$

Здесь $\varphi_{0}-$ не зависящий от координат угол поворота директора в ФН-фазе, значение которого определяется условиями минимума свободной энергии (27). Минимизация по $\varphi_{0}$ приводит к уравнению, термодинамически устойчивое решение которого $\varphi_{0}=\pi / 2$ согласно формуле (3) отвечает $\mathbf{n}=(0,1,0) \| \mathbf{H}=(0, H, 0)$, т. е. в рас-

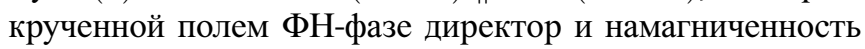
направлены по полю. Минимизация по $g_{ \pm}$дает выражения (12), в которых теперь $\varphi=\pi / 2, Q^{-1}=2 \cosh (\xi h / \kappa)$.
В точке перехода $\Phi \mathrm{X}-\Phi \mathrm{H}$, т.е. при $h=h_{c}$ свободные энергии (20) и (27) должны быть одинаковы. Это позволяет найти значение $C_{c}=2 \kappa+h_{c}^{2}$, константы $C$ при $h=h_{c}$. Подставляя теперь $C_{c}$ в уравнение $(19)$, находим уравнение для $h_{c}$

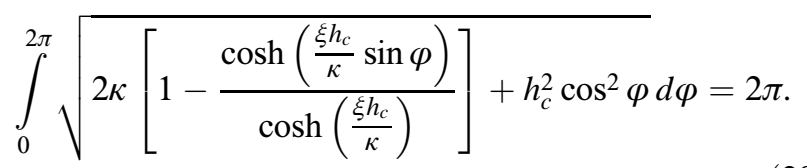

Тогда в точке перехода в ФН-фазу, т. е. при $h=h_{c}$

$$
\begin{gathered}
g_{c \pm}=Q_{c} \exp \left\{ \pm\left(\xi h_{c}\right) / \kappa\right\}, \\
Q_{c}=(1 / 2) \cosh ^{-1}\left\{\xi h_{c} / \kappa\right\}, \\
\left\langle M_{y}\right\rangle_{c}=\tanh \left\{\frac{\xi h_{c}}{\kappa}\right\} .
\end{gathered}
$$

Как видно из этих формул, при $h=h_{c}$ разность $\left(g_{c+}-g_{c-}\right) \neq 0$ и поэтому $\left\langle M_{y}\right\rangle_{c} \neq 0$, т.е. магнитные подсистемы не полностью компенсированы и упорядочены по типу ферримагнетика.

В предельном случае $\xi \ll 1$ из уравнения (28) находим $h_{c} \approx(\pi / 2)\left[1-\xi^{2} /(2 \kappa)\right]$, а при $\xi \gg 1$ поле перехода $h_{c} \approx \pi^{2} /(16 \xi)$.

Заметим, что согласно [4] уравнение для критического поля $h_{c}$ в некомпенсированном $\Phi \mathrm{X}$ - геликоидальном жидкокристаллическом ферромагнетике выглядит следующим образом:

$$
\int_{0}^{2 \pi} \sqrt{2 \kappa\left[1-\exp \left\{\frac{\xi h_{c}}{\kappa}(\sin \varphi-1)\right\}\right]+h_{c}^{2} \cos ^{2} \varphi} d \varphi=2 \pi .
$$

Чтобы определить шаг спирали и намагниченность в подкритической области $\left(h \leq h_{c}\right)$, представим уравнения для $C, Q$ и $\left\langle M_{y}\right\rangle$ в удобном для выделения асимптотических зависимостей виде. Перепишем уравнение для шага (17)

$$
C=2 \kappa+h^{2}+\frac{2 \pi}{\tilde{p}}-\frac{h^{2}}{\tilde{p}} J_{1}
$$

где

$$
J_{1}=\int_{0}^{2 \pi} \frac{\cos ^{2} \varphi d \varphi}{\sqrt{A(\varphi)}} .
$$

В уравнении (21) для $Q$ выделяем асимптотическое значение

$$
Q=\frac{\cosh ^{-1}\{\xi h / \kappa\}}{1-J_{2} / \tilde{p}},
$$

где

$$
J_{2}=\int_{0}^{2 \pi}\left(1-\exp \left\{2 \frac{\xi h}{\kappa} \cos ^{2}\left(\frac{\pi}{4}-\frac{\varphi}{2}\right)\right\}\right) \frac{d \varphi}{\sqrt{A(\varphi)}}
$$


Тогда из уравнения (26) получаем

$$
\left\langle M_{y}\right\rangle=\tanh \left\{\frac{\xi h}{\kappa}\right\}\left[\frac{1}{1-J_{2} / \tilde{p}}-\frac{2 J_{3}}{\tilde{p}}\right],
$$

где

$$
J_{3}=\int_{0}^{2 \pi} \cos ^{2}\left(\frac{\pi}{4}-\frac{\varphi}{2}\right) \exp \left\{2 \frac{\xi h}{\kappa} \cos ^{2}\left(\frac{\pi}{4}-\frac{\varphi}{2}\right)\right\} \frac{d \varphi}{\sqrt{A(\varphi)}} .
$$

Здесь интегралы $J_{1}, J_{2}, J_{3}$ остаются конечными при $h \rightarrow h_{c}$.

Из полученных формул находим, что вблизи фазового перехода $\Phi \mathrm{X}-\Phi \mathrm{H}$, т.е. при $h \rightarrow h_{c}$ зависимость шага спирали от напряженности поля выглядит следующим образом

$$
\tilde{p}=\tilde{p}_{0} \frac{h_{1}}{h_{c}-h}
$$

где

$$
h_{1}=\frac{\left(2 \pi-h_{c}^{2} J_{1 c}\right)^{2}}{4\left(\xi^{2} J_{3 c}-\kappa J_{1 c}\right)},
$$

здесь $J_{1 c}$ и $J_{3 c}-$ значения интегралов (33) и (37) при $h=h_{c}$.

Напомним, что в чистом ХЖК шаг спирали расходится вблизи перехода в нематическую фазу по логарифмическому закону [1], поэтому, как видно из (38), наличие магнитных частиц приводит к более быстрому росту шага спирали.

\section{4. Переход в ферронематическую фазу}

На рис. 1 представлена зависимость поля перехода $h_{c}$ геликоидального антиферромагнитного жидкого кристалла в ферронематическую фазу от сегрегационного параметра $\kappa$ при различных значениях параметpa $\xi$, полученная путем численного интегрирования уравнения (28). Для сравнения на рис. 2 показана зависимость $h_{c}(\kappa)$ для геликоидального ферромагнитного жидкого кристалла. Видно, что присутствие магнитной примеси приводит к понижению порога перехода по сравнению с чистым ХЖК, для которого $h_{c}=\pi / 2$. При переходе от квадрупольного режима $(\xi \ll 1$, кривые 4$)$ к дипольному ( $\xi \gg 1$, кривые 1 ) критическое поле перехода $Ф \mathrm{X}-\Phi Н$ убывает. В отличие от некомпенсированного ФХ (рис. 2), при заданном значении параметра $\xi$ компенсированный $\Phi \mathrm{X}$ (рис. 1) имеет минимум критического поля при $\kappa \approx 1$. Существование этого минимума видно из уравнения (28): при $\kappa \gg 1$ уравнение дает $h_{c} \approx(\pi / 2) /\left[1-\xi^{2} /(2 \kappa)\right]$, а при $\kappa \ll 1$ получаем $h_{c} \approx(\pi / 2) /\left[1-4 \kappa c / \pi^{2}\right]$, поэтому функция $h_{c}(\kappa)$ имеет минимум при конечных $\kappa$. Здесь $c=(1 / 2) \ln (3+2 \sqrt{2})$. Наличие минимума на рис. 1 связано с особенностями процесса сегрегации магнитной фазы. Как видно из

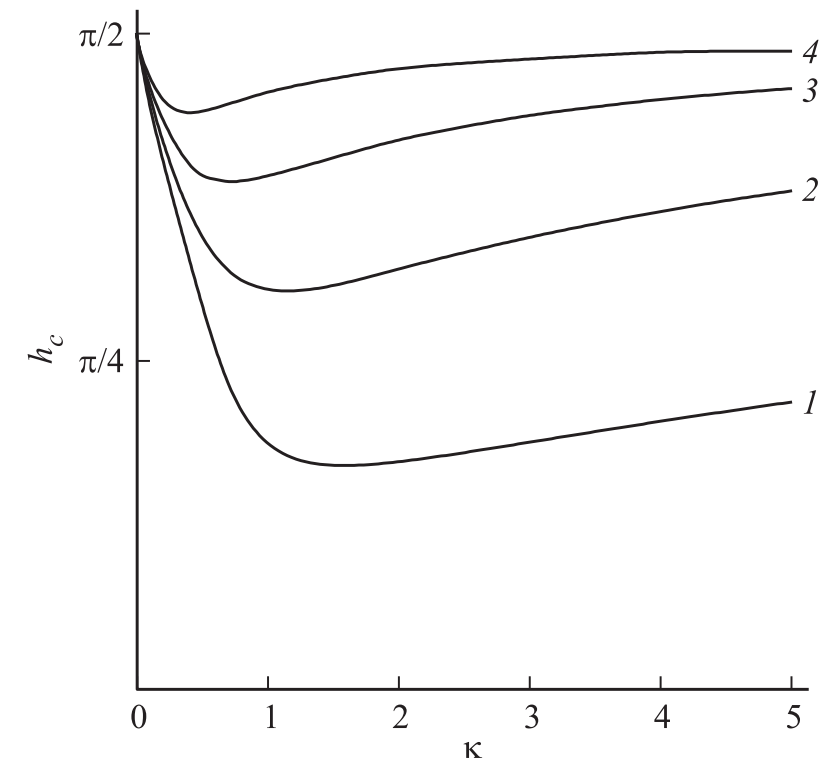

Рис. 1. Зависимость $h_{c}(\kappa)$ компенсированного ФХ от параметра $\xi$ : кривая $1-\xi=5$, кривая $2-\xi=2$, кривая $3-\xi=1$, кривая $4-\xi=0.5$.

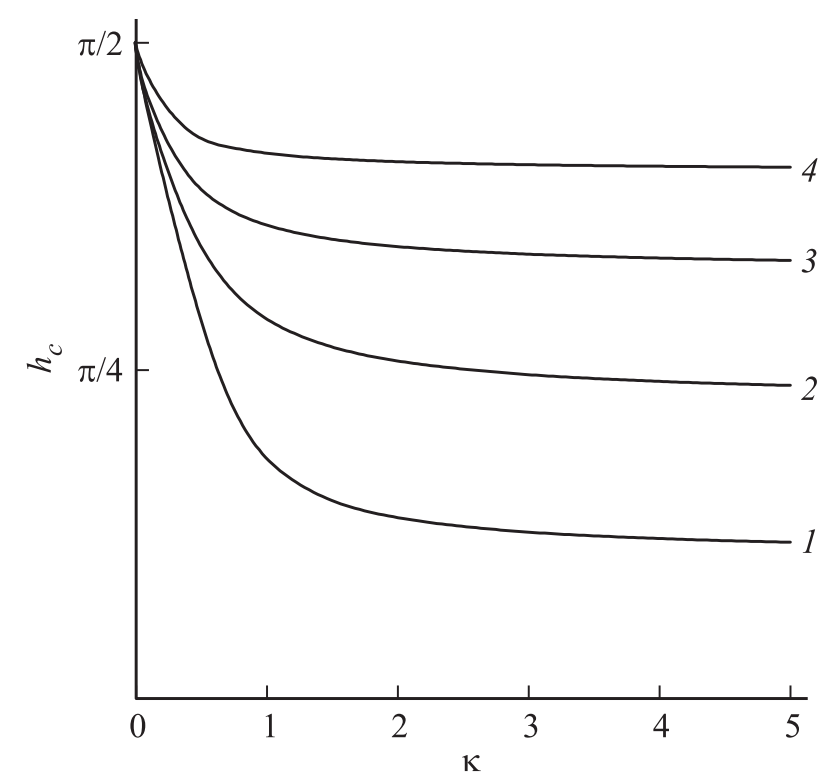

Рис. 2. Зависимость $h_{c}(\kappa)$ некомпенсированного $\Phi \mathrm{X}$ от параметра $\xi$ : кривая $1-\xi=5$, кривая $2-\xi=2$, кривая $3-\xi=1$, кривая $4-\xi=0.5$.

формулы (12), при замене $\varphi$ на $\varphi+\pi$ распределение $g_{+}$ переходит в $g_{-}$, т.е. в магнитном поле происходит миграция частиц из одной магнитной подсистемы в другую, так что частицы, „невыгодно“ ориентированные по отношению к полю в одном полувитке спирали, оказываются „выгодными“ для другого полувитка. Такого не происходит в некомпенсированном $\Phi \mathrm{X}[1]$, у которого $g \equiv g_{+}$и $g_{-} \equiv 0$, т. е. только один из полувитков спирали всегда ориентирован „выгодно“. 


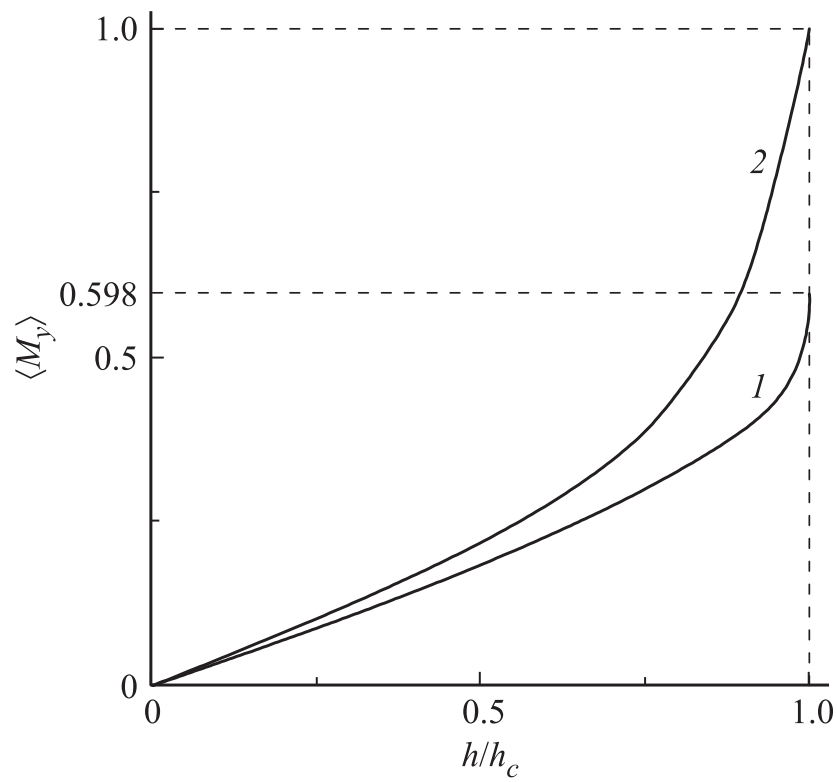

Рис. 3. Зависимость средней намагниченности компенсированного (кривая $1, h_{c}=0.687$ ) и некомпенсированного (кривая $\left.2, h_{c}=0.392\right)$ ФХ от напряженности поля при $\xi=\kappa=5$.

На рис. 3 показаны зависимости средней намагниченности $\left\langle M_{y}\right\rangle$ от напряженности поля, построенные по формуле (36). Напомним, что при $h=h_{c}$ намагниченность определяется выражением (30): это значение следует также из (36) при $h=h_{c}$, когда $p=\infty$. Как видно, в отличие от некомпенсированного ФХ [4], у которого средняя намагниченность в поле перехода $\left\langle M_{y}\right\rangle=1$ (кривая 2), в компенсированном $\Phi \mathrm{X}\left\langle M_{y}\right\rangle<1$ (кривая 1), т. е. магнитные подсистемы в точке перехода не полностью компенсированы, и ФН намагничен по типу ферримагнетика (кривая 1 на рис. 3), в то время как в некомпенсированном ФХ (кривая 2 на рис. 3) ферронематическая фаза является намагниченной по типу ферромагнетика [4].

На рис. 4 представлены зависимости шага спирали от напряженности поля для компенсированного ФХ (кривая 1) и некомпенсированного ФХ (кривая 2). В слабых полях шаг спирали растет с ростом поля согласно формулам (22) и (23), т.е. быстрее, чем в чистом ХЖК. При $h \rightarrow h_{c}$, т.е. при раскручивании спирали, шаг спирали возрастает согласно (38) и обращается в бесконечность при $h=h_{c}$. Заметим, что шаг спирали компенсированного ФХ возрастает медленнее, чем у некомпенсированного ФХ.

Как отмечалось выше, перераспределение магнитной примеси в ФХ вызывает появление средней намагниченности. На рис. 5 и 6 показаны зависимости намагниченности и шага спирали от напряженности поля для компенсированного ФХ при трех значениях параметра $\kappa$, соответствующих различным участкам кривой 1 $(\xi=5)$ на рис. $1: \kappa=0.5$ (убывающий участок), $\kappa=1.5$ (область минимума кривой) и $\kappa=5$ (возрастающий участок). Рис. 5 построен в координатах $h / h_{c}$ и приведенной намагниченности $\mu=\left\langle M_{y}\right\rangle /\left\langle M_{y}\right\rangle_{c}$. Здесь $\left\langle M_{y}\right\rangle_{c}-$ значение средней намагниченности при $h=h_{c}$, определяемое формулой (30). Из рис. 5 видно, что кривые намагничивания имеют различный характер в зависимости от значений параметра сегрегации $\kappa$. В случае

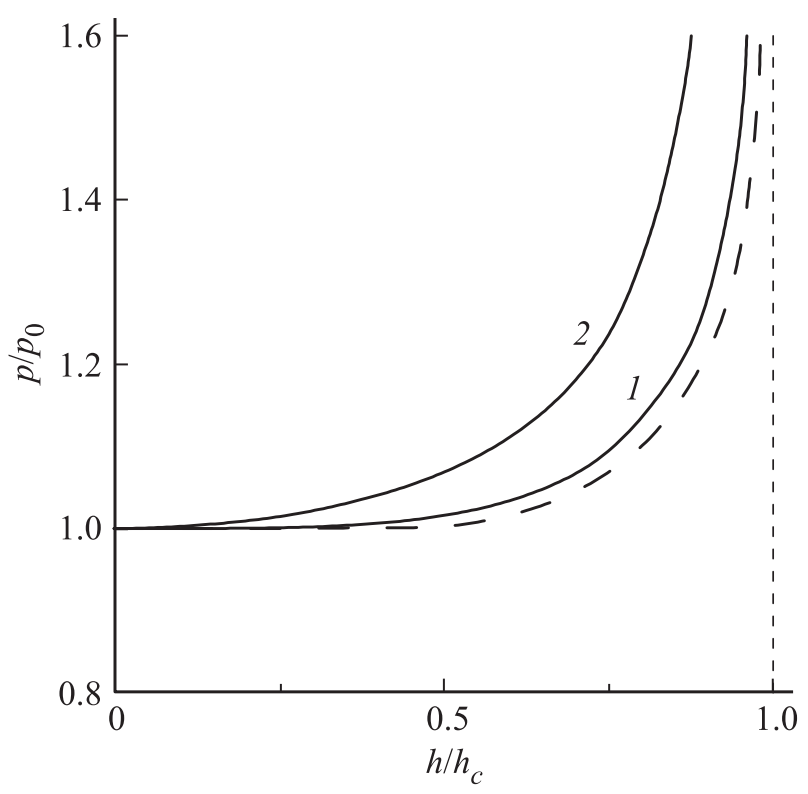

Рис. 4. Зависимость шага спирали компенсированного (кривая $1, h_{c}=0.687$ ) и некомпенсированного (кривая 2, $\left.h_{c}=0.392\right)$ ФХ от напряженности поля при $\xi=\kappa=5$, штриховая линия - для чистого ХЖК.

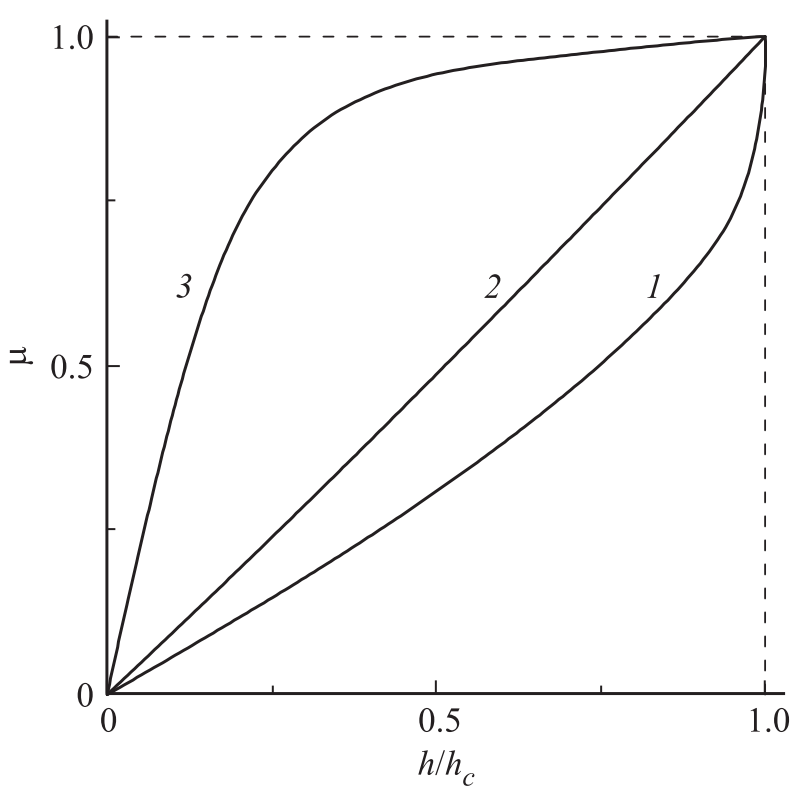

Рис. 5. Зависимость приведенной средней намагниченности $\mu=\left\langle M_{y}\right\rangle /\left\langle M_{y}\right\rangle_{c}$ компенсированного ФХ от напряженности поля для $\xi=5$. Кривая $1-\kappa=5 \quad\left(h_{c}=0.687\right)$; кривая $2-\kappa=1.5 \quad\left(h_{c}=0.530\right) ; \quad$ кривая $3-\kappa=0.5$ $\left(h_{c}=0.907\right)$. 


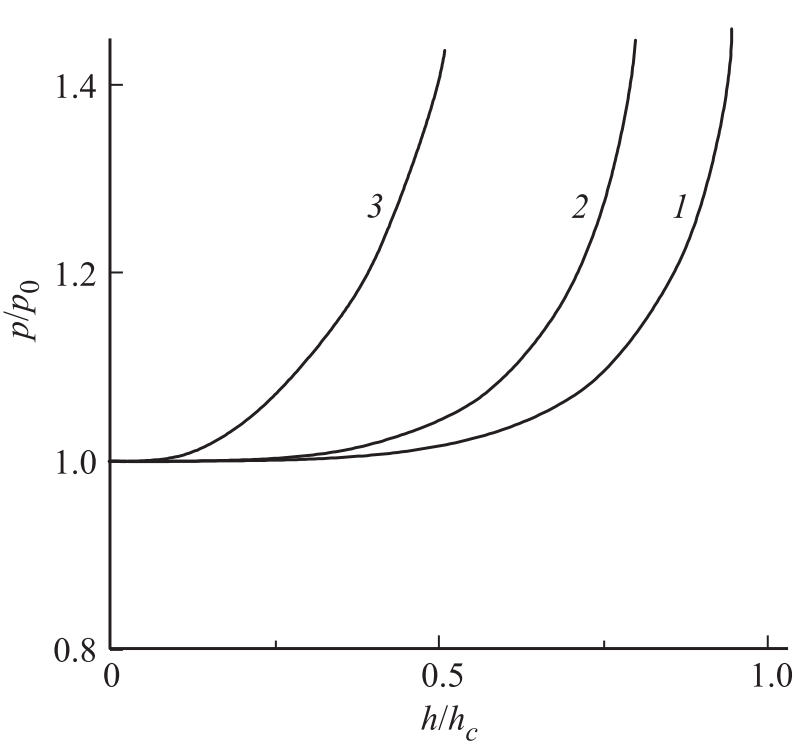

Рис. 6. Зависимость шага спирали компенсированного ФХ от напряженности поля для $\xi=5$. Кривая $1-\kappa=5\left(h_{c}=0.687\right)$; кривая $2-\kappa=1.5\left(h_{c}=0.530\right)$; кривая $3-\kappa=0.5$ $\left(h_{c}=0.907\right)$.

слабых полей $\left(h \ll h_{c}\right)$ средняя намагниченность (26) может быть представлена в виде $\left\langle M_{y}\right\rangle \approx \xi h /(2 \kappa)$, что соответствует начальному участку построенных кривых. Это позволяет найти начальную восприимчивость $\chi \approx \chi_{a} \xi^{2} /(2 \kappa)$. Таким образом, для увеличения восприимчивости ФХ необходим дипольный режим $(\xi \gg 1)$ и малые $\kappa$. Вблизи перехода в ФН-фазу, когда $\tilde{p} \gg 1$, средняя намагниченность стремится к значению (30) согласно соотношению (36).

Из рис. 6 показана зависимость шага спирали от параметра сегрегации $\kappa$ : в слабых полях шаг спирали растет с ростом поля быстрее при малых $\kappa$ согласно формуле (22), но с ростом $\kappa$ шаг медленнее расходится при $h \rightarrow h_{c}$.

\section{5. Заключение}

В настоящей работе в рамках континуального подхода изучен индуцированный магнитным полем фазовый переход геликоидального антиферромагнитного жидкого кристалла в ферримагнитную нематическую фазу. Изучено раскручивание геликоидальной структуры, получены зависимости шага спирали и намагниченности от приложенного магнитного поля и материальных параметров суспензии. Показано, что поле перехода в ферронематическую фазу существенно уменьшается в дипольном режиме раскручивания геликоидальной структуры и зависит от интенсивности сегрегации дисперсной фазы. Проанализированы отличия в поведении геликоидальных ферромагнитных и антиферромагнитных жидких кристаллов.

\section{Список литературы}

[1] П. де Жен. Физика жидких кристаллов. Мир, М. (1977).

[2] I. Abdulhalim. J. Nanophotonics 6, 061001 (2012).

[3] F. Brochard, P.G. de Gennes. J. de Phys. 31, 691 (1970).

[4] A.N. Zakhlevnykh, P.A. Sosnin. J. Magn. Magn. Mater. 146, 103 (1995).

[5] A. Zakhlevnykh, V. Shavkunov. Mol. Cryst. Liq. Cryst. 330, 593[1837] (1999).

[6] A.N. Zakhlevnykh, V.S. Shavkunov. J. Magn. Magn. Mater. 210, 279 (2000).

[7] V.S. Shavkunov, A.N. Zakhlevnykh. Mol. Cryst. Liq. Cryst. A 367, 175 (2001).

[8] W.C. Yip, H.S. Kwok. Appl. Phys. Lett. 78, 425 (2001).

[9] H. Ayeb, J. Grand, H. Sellame, S. Truong, J. Aubard, N. Felidj, A. Mlayah, E. Lacaze. J. Mater. Chem. 22, 7856 (2012).

[10] Rajaa Bitar, Gonzague Agez, M. Mitov. Soft Matter 7, 8198 (2011).

[11] B. Senyuk, M.C.M. Varney, J.A. Lopez, Sijia Wang, Ning Wuc, I. Smalyukh. Soft Matter 10, 6014 (2014).

[12] O. Kurochkin, O. Buchnev, A. Iljin, S.K. Park, S.B. Kwon, O. Grabar, Yu. Reznikov. J. Opt. A 11, 024003 (2009).

[13] N.V. Usol'tseva, M.V. Smirnova, V.V. Sotsky, A.I. Smirnova. J. Phys. Confer. ser. 558, 012003 (2014).

[14] M. Middha, R. Kumar, K.K. Raina. Liq. Cryst. 42, 1028 (2015).

[15] M. Middha, R. Kumar, K.K. Raina. AIP Conf. Proc. 1675, 030006 (2015).

[16] Qiaoxuan Zhang, P.J. Ackerman, Qingkun Liu, I.I. Smalyukh. Phys. Rev. Lett. 115, 097802 (2015).

[17] Hak Moo Lee, Hyung-Koo Chung, Hong-Gyu Park, HaeChang Jeong, Jeong-Hwan Kim, Tae-Kyu Park, Dae-Shik Seo. J. Nanosci. Nanotechnology 15, 8139 (2015).

[18] K. Stratford, A. Gray, J.S. Lintuvuori. J. Stat. Phys. 161, 1496 (2015).

[19] Ю.А. Изюмов, В.М. Лаптев. ЖЭТФ 85, 2185 (1983).

[20] Y.A. Garbovskiy, A.V. Glushchenko. Solid State Physics 62, 1 (2010).

[21] D.A. Petrov, A.N. Zakhlevnykh. Mol. Cryst. Liq. Cryst. 557, 60 (2012).

[22] A.N. Zakhlevnykh, D.A. Petrov. Phase Trans. 87, 1 (2014).

[23] A.N. Zakhlevnykh, D.A. Petrov. J. Magn. Magn. Mater. 401, 188 (2016). 